ISSN 1991-8631

\title{
Quality assessment of flour and bread from sweet potato wheat composite flour blends
}

\author{
Isaac Babatunde OLUWALANA*, Sunday Abiodun MALOMO and \\ Ezekiel Owurie OGBODOGBO \\ Department of Food Science and Technology, The Federal University of Technology, Akure, Ondo State, \\ Nigeria. \\ * Corresponding author, E-mail: ioluwalana2002@yahoo.com; Tel: +2348033976209
}

\begin{abstract}
This study was to assess the quality of the flour and bread produced from sweet potato wheat composite flour blends. Matured and freshly harvested sweet potato (Ipomea batatas L.) was obtained from a local market in Akure, Nigeria. The tubers were thoroughly washed, peeled, washed again, drained, chipped, oven dried, milled, sieved and packaged. Five blends were prepared by homogenously mixing sweet potato flour with wheat flour in the percentage proportions of: 0:100, 5:95, 10:90, 15:85 and 100:0 (PF: WF) and later used to bake bread. Chemical, microbiological and physical properties of the composite flour and bread were examined. Semi-trained Panelists were assigned to assess the bread samples as well. The results of the proximate composition showed that the protein contents of the flours ranged from 1.44-12.44\%; fat, 0.37$1.55 \%$; carbohydrate, $69.69-80.92 \%$ and the protein contents of the bread samples ranged from $10.15-12.44 \%$; fat, 4.36-4. 88\%; carbohydrate, $80.44-105.47 \%$. The flour samples had sodium content of $1.85-40.42 \mathrm{mg} / 100$ $\mathrm{g}$; calcium, 2.71-17.14 mg/ $100 \mathrm{~g}$; potassium, $160.02-175.12 \mathrm{mg} / 100 \mathrm{~g}$; selenium, 0.006-0.620 mg/ $100 \mathrm{~g}$, while bread samples had sodium, $46.86-550.77 \mathrm{mg} / 100 \mathrm{~g}$; calcium, 3.59-6.63 mg/ $100 \mathrm{~g}$; potassium, 162.42$388.75 \mathrm{mg} / 100 \mathrm{~g}$; selenium, 0.19-0.27 mg/ $100 \mathrm{~g}$. Pasting properties showed that flour had breakdown (38.3360.76 RVU) and setback values (82.86-173.50 RVU). The microbiological results of the flour ranged from $1.0 \times 10^{3}$ to $7.0 \times 10^{3} \mathrm{cfu} / \mathrm{g}$ (bacteria) and $1.0 \times 10^{4}$ to $8.0 \times 10^{4} \mathrm{sfu} / \mathrm{g}$ (fungi) while for bread, $1.0 \times 10^{3}$ to $4.0 \times 10^{3}$ $\mathrm{cfu} / \mathrm{g}$ (bacteria) and $1.0 \times 10^{4}$ to $8.0 \times 10^{4} \mathrm{sfu} / \mathrm{g}\left(4.5 \times 10^{4}\right)$. No visible growth was recorded for E. coli. and Coliform bacteria. The bread loaf weights ranged from 450 to $500 \mathrm{~g}$; loaf volume, $1171.73-1239.84 \mathrm{~cm}^{3}$ and specific loaf volume, $2.48-2.62 \mathrm{~cm}^{3} / \mathrm{g}$. There is no significant difference in the crust colour, crumb holes, stability, elasticity, firmness, shape regularity and appearance of the samples. No panelist showed a total dislike for the taste of any of the samples. All the qualities evaluated did not significantly $(\mathrm{p} \leq 0.05)$ affect the acceptability and preference of the samples. Hence, it was concluded that wheat flour could be substituted with sweet potato flour up to $15 \%$ level in bread making which will still retain much of the physicochemical and sensory properties.
\end{abstract}

() 2012 International Formulae Group. All rights reserved.

Keywords: Panelists, Pasting, Microbiological, Nutritional and Sensory properties.

\section{INTRODUCTION}

Bread, the major bakery product of cereal grain, is a staple food in several cultures (Malomo et al., 2011). It is so important in human diet that increases in its price have triggered off angry protests in some

(C) 2012 International Formulae Group. All rights reserved.

DOI : http://dx.doi.org/10.4314/ijbcs.v6i1.6 
countries where up to $50 \%$ of their total calories are supplied by bread alone (Pomeranz and Clifton, 1996; Akobundu, 2006). Even in a country like Nigeria where starchy foods constitute significant calorie intake, bread consumption has increased tremendously in recent times. For instance, Akobundu (2006) reported that with increasing population and cravings for fast and convenience foods now, bread consumption in the society has greatly increased.

Bread, a steady and important staple food in Nigeria with increasing consumption rate, is however, relatively expensive according to the reports of Edema et al. (2005) and Olaoye et al. (2006). This is because the major raw material in bread making, wheat, is an imported and temperate cereal crop, which may not grow in the tropics due to climatic reasons. With this progressive increase in the bread consumption in Nigeria, efforts have now been made to promote the use of composite flours in which flours from locally and indigenous grown crops and high protein seeds were partially substituted into wheat flour for bread making. This composite flour programme would thereby minimize the demand for imported wheat; produce proteinenriched bread (Giami et al., 2004; Olaoye et al., 2006); conserve foreign reserves (Eddy et al., 2007) and widen the utilization of indigenous crops in food formulation (AdeOmowaye et al., 2008).

Reports have been published on the successful composite bread technology (though such bread still require at least $70 \%$ wheat flour to be able to rise) using some indigenous crops like soybeans, plantain, cocoyam, sweet potato, breadfruit, breadnut etc (Oluwole et al., 2005; Onuh and Egwujeh, 2005; Olaoye et al. 2006; Eddy et al., 2007; Ade-Omowaye et al., 2008; Malomo, 2010; Malomo et al., 2011). Iheagwara and Iwuoha (2005) described sweet potato (Ipomea batatas L.) as an important staple food crop which is eaten as fresh boiled roots and traditionally processed into low quality flour for domestic use. Oluwole et al. (2005) reported that I. batatas L. is often grown with other crops such as maize and its consumer's preference is low because of its sugary content after boiling. He further opined that it could be consumed with vegetables and soup; fried as chips for snack production and as well processed into flour for making bread loaves, cookies, and possibly as breakfast meal. Onuh and Egwujeh (2005) reported that sweet potato flour stores fairly well for months, especially under low moisture conditions and water activity which greatly caused a significant reduction in the number of spoilage microorganisms with no significant changes in its colour, texture and acceptability.

The objective of this work is to assess the quality of the flour and the baking qualities of the bread produced from sweet potato- wheat composite flour.

\section{MATERIALS AND METHODS Materials}

Mature, insect free and freshly harvested sweet potato (Ipomea batatas L.) tubers were purchased from a local market in Akure, Nigeria. Commercial hard wheat flour (100\%), baking yeast (DSM bakery ingredient, Dordrecht, Holland), baking fat (PT Intiboga Sejahtera, Jakarta, Indonesia), sugar (Dangote Groups, Lagos Nigeria), improver (Vitamin C), water and salt were purchased from a baking ingredients store at Akure, Nigeria. Kenwood mixer (Model A 907 D, Kenwood Ltd, England), electric oven (SL- 9 Infrared Food Oven, Hubert, China) and other baking equipment of The Federal University of Technology, Akure (FUTA) bakery unit were used.

\section{Methods \\ Production of sweet potato flour}

Fresh sweet potato (Ipomea batatas L.) tubers were thoroughly washed, peeled, washed again, drained, chipped, oven dried in laboratory oven (LCON53CF, Genlab, England), (105 $\left.{ }^{\circ} \mathrm{C}, 1-3 \mathrm{~h}\right)$, milled, sieved 
(0.35 mm mesh size) and packaged in well labeled flour sack until required for composite flour preparation.

\section{Composite flour blend formulation}

Five blends were prepared by homogenously mixing sweet potato flour with wheat flour in the percentage proportions: 0:100, 5:95, 10:90, 15:85 and 100:0 (PF: WF) using a Kenwood food processor (Model A 907 D, Kenwood Ltd, England).

\section{Bread making}

Bread was produced using the straight dough process. Baking trials were carried out under laboratory conditions to optimize baking conditions prior to the actual runs. Flour and dough weighing were carried out on laboratory-scale equipment (CE- 410I, Camry Emperors, China). Dough was mixed to optimum consistency in a Kenwood mixer (Model A 907 D, Kenwood Ltd, England) with low speed of $85 \mathrm{rpm}$ for $1 \mathrm{~min}$. Final dough temperature was $30{ }^{\circ} \mathrm{C}$. Dough rested in bulk for $45 \mathrm{~min}$, kneaded and left to proof for $15 \mathrm{~min}$. After proofing the dough was scaled into $500 \mathrm{~g}$ portions, manually rounded, rolled, put in tin baking pans $\left(75 \mathrm{~min}, 30{ }^{\circ} \mathrm{C}, 80 \%\right.$ relative humidity). Baking was done at 230 ${ }^{\circ} \mathrm{C}$ in an electric oven (Electric oven SL- 9 Infra red Food Oven, Hubert, China) until the golden brown colour was formed. The resulting bread samples were allowed to cool to room temperature $\left(37{ }^{\circ} \mathrm{C}\right)$ for $2 \mathrm{~h}$ before further analysis was carried out.

\section{Analysis}

Determination of pasting properties of composite flours

The pasting properties of the composite flours were studied using a Rapid Visco Analyzer (RVA). Sixty five grams of the substituted flours were homogeneously dispersed in $450 \mathrm{ml}$ distilled water. The suspension was heated from 30 to $95{ }^{\circ} \mathrm{C}$ (at $1.5{ }^{\circ} \mathrm{C} / \mathrm{min}$ ), kept at $95{ }^{\circ} \mathrm{C}$ for $15 \mathrm{~min}$ and cooled to $50{ }^{\circ} \mathrm{C}$ (at $1.5{ }^{\circ} \mathrm{C} / \mathrm{min}$ ). Finally, the paste was held at $50{ }^{\circ} \mathrm{C}$ for $15 \mathrm{~min}$. Peak viscosity and time, pasting temperatures, final viscosity and trough were read off the RVA.
Setback and breakdown were then calculated and expressed as RVU.

\section{Chemical composition of composite flours and bread samples \\ Proximate composition}

The proximate composition of the composite flours and bread samples were determined as described by AOAC (1990) methods 14.091, 14.103, 14.093, 14.111 and 14.108 , respectively. The nitrogen conversion factor used for crude protein calculation was 5.70. The carbohydrate content was obtained by difference. For each type of bread, three loaves were frozen, dried and ground into a coarse powder (60 mesh size) before analysis.

\section{Mineral composition}

Mineral composition namely: $\mathrm{Na}, \mathrm{K}$, $\mathrm{Ca}, \mathrm{Mg}, \mathrm{Fe}, \mathrm{Cu}, \mathrm{Zn}, \mathrm{Mn}, \mathrm{P}$ and $\mathrm{Se}$ were determined by wet ashing method (AOAC, 1990). The Atomic Absorption Spectrometer (Perkin-Elmer Model 403, Norwalk CT, USA) was used to determine the concentrations of the metals in the samples by flame atomization, using air acetylene flame and single element hollow cathode lamp and following the equipment procedures (AOAC, 1990). Phosphorus was determined using vanadomolybdate colorimetric method (AOAC, 1990).

\section{Microbiological studies of flour and bread}

The aerobic plate count was carried out on the flour and bread samples according to methods of AOAC (1990). Serial dilution and pour plate techniques were used. Nutrient agar (NA) was used for bacteria while Eosin methylene blue (EMB) was used for fungi culturing. Viable colonies count was recorded for bacteria as colony forming units (cfu/g) and spore forming units (sfu/g) for fungi. .

Evaluation of the baking qualities of bread samples

Loaf weight: The loaf weight was determined by weighing the bread loaves 20 mins. after baking, using the laboratory scale (CE- 410I, Camry Emperors, China) and the readings recorded in grammes. 
Loaf volume: The loaf volume was determined by using Rape seed displacement method (AACC, 2000, Standard 10-05). This was done by loading sorghum grains into an empty box with calibrated mark until it reached the marked level and unloaded back. The bread sample was put into the box and the measured sorghum was loaded back again. The remaining sorghum grains left outside the box was measured using measuring cylinder and recorded as loaf volume in $\mathrm{cm}^{3}$.

Specific volume: The specific volume (volume to mass ratio) $\left(\mathrm{cm}^{3} / \mathrm{g}\right)$ was thereafter calculated.

\section{Other baking qualities}

Bread height and diameter were measured by using a measuring ruler and the loaf shape (height to diameter ratio) was also calculated from these values. The crumb and crust colour, crumb holes, stability, crumbling, surface resistance to light of the bread samples were also evaluated after sufficient cooling. The reflective surface colour of bread samples were measured by changing the surfaces of the bread towards light rays.

\section{Sensory evaluation}

The following sensory attributes, viz: taste, aroma, texture, appearance and overall acceptability, were assessed on bread samples from the composite flours and the control within 3 to $6 \mathrm{~h}$ of baking using 9- point hedonic scale.

\section{Statistical analysis}

All determinations were performed in triplicate. The statistical analyses were conducted using one-way ANOVA procedures. Statistical differences in samples were tested for at $p<0.05$. Duncan's New Multiple-Range Test (DMRT) was used to separate the mean values. All the analyses were done with SPSS (11.0) software.

\section{RESULTS}

The proximate composition of flours and bread samples are presented in Tables 1 and 2 respectively. The protein content of the flours ranged from $1.44-12.44 \%$; fat, 0.37 1.55\%; ash, 1.18-2.88\%; fibre, 2.36- $2.75 \%$; moisture 10.77-12.52\%; carbohydrate, 69.69$80.92 \%$. From table 2, the protein content of the bread samples ranged from $10.15-12.44 \%$; fat, 4.36-4. 88\%; ash, 1.18- 2.78\%; fibre, 2.58-2.75\%; moisture, $38.76-45.66 \%$; carbohydrate, $80.44-105.47 \%$.

The mineral composition of flours and bread samples are presented in Table 3 and 4 respectively. The results show that flour had $1.85-40.42 \mathrm{mg} / 100 \mathrm{~g}$ of sodium $(\mathrm{Na})$ with $100 \%$ PF having the highest and also highest in $\mathrm{K}, \mathrm{P}$, and $\mathrm{Cu}$ than the control (100\% WF). The calcium $(\mathrm{Ca})$ content ranged from 2.71$17.14 \mathrm{mg} / 100 \mathrm{~g}$; potassium (K), 160.02$175.12 \mathrm{mg} / 100 \mathrm{~g}$; selenium (Se), 0.006-0.620 $\mathrm{mg} / 100 \mathrm{~g}$. Table 4 shows that bread samples had sodium (Na), 46.86-550.77 mg/100 g with bread sample $15 \% \mathrm{PF} / 85 \%$ WF having the highest value in $\mathrm{Na}$ and $\mathrm{P}$ than the control. The calcium $(\mathrm{Ca})$ content ranged from 3.59$6.63 \mathrm{mg} / 100 \mathrm{~g}$; potassium (K), 162.42-388.75 $\mathrm{mg} / 100 \mathrm{~g}$; selenium (Se), 0.19-0.27 mg/100 g.

Table 5 shows the effect of substitution of wheat flour with sweet potato flour on its pasting properties. Peak (206.75-301.08 RVU), breakdown (38.33-60.76 RVU), final viscousities (130.83-413.83 RVU) and setback values (82.86-173.50 RVU) of the substituted flours were significantly lower than the control sample (315.50, 78.58, 420.67 and 183.75 RVU respectively).

The results of the microbiological studies carried out on the composite flours and bread samples are presented in Tables 6 and 7. Table 6 shows that the bacteria count of the flour had $0 \% \mathrm{PF} / 100 \% \mathrm{WF}\left(1.0 \times 10^{3} \mathrm{cfu} / \mathrm{g}\right), 100 \%$ $\mathrm{PF} / 0 \% \mathrm{WF}\left(4.0 \times 10^{3} \mathrm{cfu} / \mathrm{g}\right), 5 \% \mathrm{PF} / 95 \% \mathrm{WF}$ $\left(7.0 \times 10^{3} \mathrm{cfu} / \mathrm{g}\right), 10 \% \mathrm{PF} / 90 \% \mathrm{WF}\left(5.0 \times 10^{3}\right.$ $\mathrm{cfu} / \mathrm{g})$ and $15 \% \mathrm{PF} / 85 \% \mathrm{WF}\left(3.0 \times 10^{3} \mathrm{cfu} / \mathrm{g}\right)$ while fungi growth were $1.0 \times 10^{4} \mathrm{sfu} / \mathrm{g}$, $8.0 \times 10^{4} \mathrm{sfu} / \mathrm{g}, 3.0 \times 10^{4} \mathrm{sfu} / \mathrm{g}, 4.0 \times 10^{4} \mathrm{sfu} / \mathrm{g}$ and $2.0 \times 10^{4} \mathrm{sfu} / \mathrm{g}$ respectively. Table 7 shows that the bacteria count for bread samples are $0: 100\left(4.0 \times 10^{3} \mathrm{cfu} / \mathrm{g}\right), 5: 95\left(2.0 \times 10^{3} \mathrm{cfu} / \mathrm{g}\right)$, $10: 90\left(1.0 \times 10^{3} \mathrm{cfu} / \mathrm{g}\right), 15: 85\left(1.0 \times 10^{3} \mathrm{cfu} / \mathrm{g}\right)$ 
while fungi growth were $1.0 \times 10^{4} \mathrm{sfu} / \mathrm{g}$, $8.0 \times 10^{4} \mathrm{sfu} / \mathrm{g}, 2.0 \times 10^{4} \mathrm{sfu} / \mathrm{g}$, and $7.0 \times 10^{4}$ $\mathrm{sfu} / \mathrm{g}$ respectively.

From Table 8, sample 10:90 has the best loaf shape when compared with the control while bread at 5\% level of substitution has the least. Their loaf weights ranged from 450-500 g; loaf volume, $1171.73-1239.84 \mathrm{~cm}^{3}$ and specific loaf volume, $2.48-2.62 \mathrm{~cm}^{3} / \mathrm{g}$. There is no significant difference between the loaf weights of the samples substituted at $5 \%$ and $10 \%$.

The result of the quality and sensory evaluation of the bread samples are presented in Tables 9 and 10. The panelists showed preferences for all the samples presented to them and there is no wide significant differences when compared with the control sample.

Table 1: Proximate composition of flour samples (\%).

\begin{tabular}{lcccccc}
\hline Samples & Protein & Fat & Ash & Fibre & Moisture & Carbohydrate \\
\hline $0: 100$ & $12.44 \mathrm{a} \pm 0.01$ & $1.55 \mathrm{a} \pm 0.01$ & $1.22 \mathrm{~b} \pm 0.01$ & $2.36 \mathrm{~b} \pm 0.01$ & $12.52 \mathrm{a} \pm 0.19$ & $69.69 \mathrm{e} \pm 0.03$ \\
$5: 95$ & $11.62 \mathrm{~b} \pm 0.01$ & $1.50 \mathrm{a} \mathrm{b} \pm 0.05$ & $2.88 \mathrm{a} \pm 0.05$ & $2.40 \mathrm{~b} \pm 0.01$ & $12.05 \mathrm{~b} \pm 0.02$ & $71.38 \mathrm{~d} \pm 0.05$ \\
$10: 90$ & $10.34 \mathrm{c} \pm 0.02$ & $1.45 \mathrm{~b} \pm 0.01$ & $2.84 \mathrm{a} \pm 0.02$ & $2.46 \mathrm{~b} \pm 0.01$ & $11.84 \mathrm{bc} \pm 0.02$ & $72.74 \mathrm{c} \pm 0.02$ \\
$15: 85$ & $8.77 \mathrm{~d} \pm 0.01$ & $1.42 \mathrm{~b} \pm 0.01$ & $2.87 \mathrm{a} \pm 0.00$ & $2.49 \mathrm{~b} \pm 0.05$ & $11.05 \mathrm{c} \pm 0.02$ & $74.65 \mathrm{~b} \pm 0.01$ \\
$100: 0$ & $1.44 \mathrm{e} \pm 0.05$ & $0.37 \mathrm{c} \pm 0.01$ & $1.18 \mathrm{~b} \pm 0.05$ & $2.75 \mathrm{a} \pm 0.01$ & $10.77 \mathrm{~d} \pm 0.02$ & $80.92 \mathrm{a} \pm 0.01$ \\
& & & & & & \\
\hline
\end{tabular}

Means followed by the same letter of alphabets in a column are not significantly different at $\mathrm{p} \leq 0.05$.

Values are means of triplicate readings.

$0: 100=0 \% \mathrm{PF} / 100 \% \mathrm{WF}$,

$5: 95=5 \% \mathrm{PF} / 95 \% \mathrm{WF}$,

$10: 90=10 \% \mathrm{PF} / 90 \% \mathrm{WF}$,

$15: 85=15 \% \mathrm{PF} / 85 \% \mathrm{WF}$;

100:0 $=100 \% \mathrm{PF} / 0 \% \mathrm{WF}$

Table 2: Proximate composition of bread samples (\%).

\begin{tabular}{lcccccc}
\hline Samples & Protein & Fat & Ash & Fibre & Moisture & Carbohydrate \\
\hline $0: 100$ & $12.44 \mathrm{a} \pm 0.06$ & $4.36 \mathrm{~d} \pm 0.06$ & $1.18 \mathrm{~d} \pm 0.00$ & $2.75 \mathrm{a} \pm 0.01$ & $38.76 \mathrm{~d} \pm 0.01$ & $80.44 \mathrm{~d} \pm 0.02$ \\
$5: 95$ & $11.66 \mathrm{~b} \pm 0.06$ & $4.88 \mathrm{a} \pm 0.01$ & $2.78 \mathrm{a} \pm 0.06$ & $2.58 \mathrm{~b} \pm 0.01$ & $45.66 \mathrm{a} \pm 0.01$ & $91.48 \mathrm{c} \pm 0.01$ \\
$10: 90$ & $11.28 \mathrm{c} \pm 0.06$ & $4.68 \mathrm{~b} \pm 0.06$ & $2.65 \mathrm{ab} \pm 0.01$ & $2.63 \mathrm{~b} \pm 0.06$ & $43.53 \mathrm{c} \pm 0.01$ & $99.88 \mathrm{~b} \pm 0.06$ \\
$15: 85$ & $10.15 \mathrm{~d} \pm 0.02$ & $4.77 \mathrm{~b} \pm 0.01$ & $2.54 \mathrm{c} \pm 0.06$ & $2.66 \mathrm{~b} \pm 0.06$ & $44.38 \mathrm{~b} \pm 0.01$ & $105.47 \mathrm{a} \pm 0.06$ \\
\hline
\end{tabular}

Means followed by the same letter of alphabets in a column are not significantly different at $p \leq 0.05$.

Values are means of triplicate readings.

$0: 100=0 \% \mathrm{PF} / 100 \% \mathrm{WF}$.

$5: 95=5 \% \mathrm{PF} / 95 \% \mathrm{WF}$,

$10: 90=10 \% \mathrm{PF} / 90 \% \mathrm{WF}$,

$15: 85=15 \% \mathrm{PF} / 85 \% \mathrm{WF}$;

$100: 0=100 \% \mathrm{PF} / 0 \% \mathrm{WF}$. 
Table 3: Mineral composition of flour samples (mg/100g).

\begin{tabular}{lcccccccccc}
\hline Samples & $\mathbf{C a}$ & $\mathbf{F e}$ & $\mathbf{Z n}$ & $\mathbf{C u}$ & $\mathbf{M g}$ & $\mathbf{P}$ & $\mathbf{K}$ & $\mathbf{M n}$ & $\mathbf{\text { Se }}$ \\
\hline $0: 100$ & $17.14 \mathrm{a} \pm 0.02$ & $1.12 \mathrm{a} \pm 0.03$ & $0.66 \mathrm{~d} \pm 0.01$ & $0.112 \mathrm{~b} \pm 0.00$ & $24.66 \mathrm{~b} \pm 0.00$ & $117.11 \mathrm{e} \pm 0.01$ & $160.02 \mathrm{e} \pm 0.01$ & $1.85 \mathrm{e} \pm 0.01$ & $0.42 \mathrm{~b} \pm 0.01$ & $0.620 \mathrm{a} \pm 0.00$ \\
$5: 95$ & $14.52 \mathrm{~b} \pm 0.01$ & $1.05 \mathrm{~b} \pm 0.03$ & $0.74 \mathrm{~b} \pm 0.00$ & $1.114 \mathrm{~b} \pm 0.00$ & $24.77 \mathrm{a} \pm 0.04$ & $118.42 \mathrm{~d} \pm 0.01$ & $161.02 \mathrm{~d} \pm 0.01$ & $1.92 \mathrm{~d} \pm 0.01$ & $0.55 \mathrm{a} \pm 0.03$ & $0.520 \mathrm{~b} \pm 0.00$ \\
$10: 90$ & $12.55 \mathrm{c} \pm 0.03$ & $1.02 \mathrm{bc} \pm 0.02$ & $0.78 \mathrm{ab} \pm 0.00$ & $0.116 \mathrm{ab} \pm 0.00$ & $24.62 \mathrm{~b} \pm 0.01$ & $118.82 \mathrm{c} \pm 0.01$ & $163.33 \mathrm{c} \pm 0.02$ & $2.02 \mathrm{c} \pm 0.01$ & $0.59 \mathrm{a} \pm 0.03$ & $0.42 \mathrm{c} \pm 0.00$ \\
$15: 85$ & $11.63 \mathrm{~d} \pm 0.04$ & $0.98 \mathrm{c} \pm 0.02$ & $0.81 \mathrm{a} \pm 0.00$ & $0.117 \mathrm{ab} \pm 0.00$ & $24.42 \mathrm{c} \pm 0.01$ & $119.22 \mathrm{~b} \pm 0.01$ & $164.17 \mathrm{~b} \pm 0.04$ & $2.15 \mathrm{~b} \pm 0.03$ & $0.61 \mathrm{a} \pm 0.01$ & $0.28 \mathrm{~d} \pm 0.00$ \\
$100: 0$ & $2.71 \mathrm{e} \pm 0.04$ & $0.33 \mathrm{~d} \pm 0.02$ & $0.21 \mathrm{~d} \pm 0.00$ & $0.126 \mathrm{a} \pm 0.00$ & $24.44 \mathrm{c} \pm 0.02$ & $119.88 \mathrm{a} \pm 0.05$ & $175.12 \mathrm{a} \pm 0.01$ & $40.42 \mathrm{a} \pm 0.01$ & $0.38 \mathrm{~b} \pm 0.05$ & $0.06 \mathrm{e} \pm 0.00$ \\
\hline
\end{tabular}

Means followed by the same letter of alphabets in a column are not significantly different at $p \leq 0.05$.

Values are means of triplicate readings.

$0: 100=0 \% \mathrm{PF} / 100 \% \mathrm{WF}$

$5: 95=5 \% \mathrm{PF} / 95 \% \mathrm{WF}$,

$10: 90=10 \% \mathrm{PF} / 90 \% \mathrm{WF}$,
$15: 85=15 \% \mathrm{PF} / 85 \% \mathrm{WF}$

$15: 85=15 \%$ PF $/ 85 \% \mathrm{WF}$

$100: 0=100 \% \mathrm{PF} / 0 \% \mathrm{WF}$

Table 4: Mineral composition of bread samples (mg/100g).

\begin{tabular}{|c|c|c|c|c|c|c|c|c|c|c|}
\hline Samples & $\mathbf{C a}$ & $\mathbf{F e}$ & $\mathbf{Z n}$ & $\mathbf{C u}$ & Mg & $\mathbf{P}$ & $\mathbf{K}$ & $\mathbf{N a}$ & Mn & Se \\
\hline $0: 100$ & $3.59 \mathrm{~d} \pm 0.05$ & $0.35 \mathrm{c} \pm 0.03$ & $0.21 \mathrm{c} \pm 0.00$ & $0.13 \pm 0.00$ & $26.33 a \pm 0.02$ & $122.22 b \pm 0.01$ & $388.75 a \pm 0.03$ & $46.86 \mathrm{~d} \pm 0.03$ & $0.22 \mathrm{c} \pm 0.01$ & $0.27 \mathrm{a} \pm 0.00$ \\
\hline $5: 95$ & $6.63 a \pm 0.02$ & $0.72 \mathrm{a} \pm 0.01$ & $1.12 b \pm 0.01$ & $0.12 \pm 0.00$ & $22.12 d \pm 0.01$ & $133.33 c \pm 0.01$ & $162.42 \mathrm{~d} \pm 0.01$ & $547.22 b \pm 0.01$ & $0.63 a \pm 0.01$ & $0.25 \mathrm{a} \pm 0.00$ \\
\hline 10:90 & $5.42 \mathrm{~b} \pm 0.01$ & $0.63 \mathrm{ab} \pm 0.02$ & $1.28 \mathrm{a} \pm 0.05$ & $0.12 \pm 0.00$ & $24.12 \mathrm{c} \pm 0.01$ & $142.12 b \pm 0.01$ & $165.22 \mathrm{~b} \pm 0.01$ & $525.02 \mathrm{c} \pm 0.01$ & $0.55 b \pm 0.03$ & $0.20 \mathrm{~b} \pm 0.00$ \\
\hline $15: 85$ & $5.28 \mathrm{c} \pm 0.01$ & $0.58 \mathrm{~b} \pm 0.05$ & $1.26 \mathrm{a} \pm 0.03$ & $0.11 \pm 0.00$ & $24.42 b \pm 0.01$ & $148.18 \mathrm{a} \pm 0.05$ & $16.22 \mathrm{c} \pm 0.01$ & $550.77 \mathrm{a} \pm 0.04$ & $0.59 \mathrm{ab} \pm 0.05$ & $0.19 b \pm 0.00$ \\
\hline
\end{tabular}

Means followed by the same letter of alphabets in a column are not significantly different at $p \leq 0.05$.

Values are means of triplicate readings.

$0: 100=0 \% \mathrm{PF} / 100 \% \mathrm{WF}$

$5: 95=5 \% \mathrm{PF} / 95 \% \mathrm{WF}$

$10: 90=10 \% \mathrm{PF} / 90 \% \mathrm{WF}$,
$15: 85=15 \% \mathrm{PF} / 85 \% \mathrm{WF}$. 
Table 5: Pasting properties of flour samples.

\begin{tabular}{lccccccc}
\hline Samples & $\begin{array}{c}\text { Peak } \\
\text { Viscosity }(\mathbf{R V U})\end{array}$ & $\begin{array}{c}\text { Trough } \\
(\mathbf{R V U})\end{array}$ & $\begin{array}{c}\text { Breakdown } \\
(\mathbf{R V U})\end{array}$ & $\begin{array}{c}\text { Final Viscosity } \\
(\mathbf{R V U})\end{array}$ & $\begin{array}{c}\text { Setback } \\
(\mathbf{R V U})\end{array}$ & $\begin{array}{c}\text { Peak Time } \\
(\mathbf{m i n})\end{array}$ & $\begin{array}{c}\text { Pasting Temperature } \\
\left({ }^{\mathbf{0}} \mathbf{C}\right)\end{array}$ \\
\hline $0: 100$ & $315.50 \mathrm{a} \pm 0.17$ & $236.92 \mathrm{~b} \pm 0.01$ & $78.58 \mathrm{a} \pm 0.57$ & $420.67 \mathrm{a} \pm 0.01$ & $183.75 \mathrm{a} \pm 0.01$ & $4.80 \mathrm{e} \pm 0.05$ & $84.90 \mathrm{e} \pm 0.05$ \\
$5: 95$ & $301.08 \mathrm{~b} \pm 0.57$ & $240.33 \mathrm{a} \pm 0.17$ & $60.76 \mathrm{~b} \pm 0.04$ & $413.83 \mathrm{~b} \pm 0.05$ & $173.50 \mathrm{~b} \pm 0.05$ & $4.93 \mathrm{~d} \pm 0.01$ & $86.85 \mathrm{~d} \pm 0.05$ \\
$10: 90$ & $239.25 \mathrm{c} \pm 0.11$ & $133.10 \mathrm{c} \pm 0.10$ & $58.11 \mathrm{c} \pm 0.05$ & $254.91 \mathrm{c} \pm 0.05$ & $156.68 \mathrm{~d} \pm 0.15$ & $5.01 \mathrm{c} \pm 0.05$ & $90.008 \mathrm{c} \pm 0.05$ \\
$15: 85$ & $206.75 \mathrm{~d} \pm 1.13$ & $128.58 \mathrm{~d} \pm 0.57$ & $52.84 \mathrm{~d} \pm 0.33$ & $191.28 \mathrm{~d} \pm 0.08$ & $162.67 \mathrm{c} \pm 0.01$ & $5.64 \mathrm{~b} \pm 0.05$ & $95.25 \mathrm{~b} \pm 0.01$ \\
$100: 0$ & $239.25 \mathrm{c} \pm 0.11$ & $94.83 \mathrm{e} \pm 0.10$ & $38.33 \mathrm{e} \pm 0.05$ & $130.83 \mathrm{e} \pm 0.05$ & $82.86 \mathrm{e} \pm 0.15$ & $5.88 \mathrm{a} \pm 0.05$ & $98.76 \mathrm{a} \pm 0.05$ \\
\hline
\end{tabular}

Means followed by the same letter of alphabets in a column are not significantly different at $\mathrm{p} \leq 0.05$.

$0: 100=0 \% \mathrm{PF} / 100 \% \mathrm{WF}$,

$5: 95=5 \% \mathrm{PF} / 95 \% \mathrm{WF}$,

$10: 90=10 \% \mathrm{PF} / 90 \% \mathrm{WF}$,

$15: 85=15 \% \mathrm{PF} / 85 \% \mathrm{WF}$

$100: 0=100 \% \mathrm{PF} / 0 \% \mathrm{WF}$.

Table 6: Aerobic count of viable organisms of flour samples.

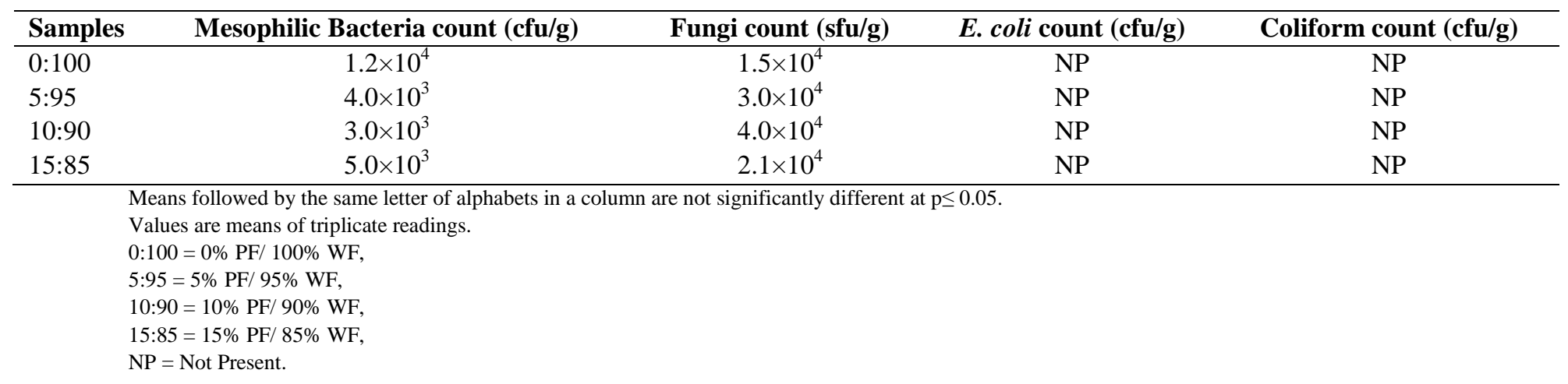


Table 7: Aerobic count of viable organisms of bread samples.

\begin{tabular}{|c|c|c|c|c|}
\hline Samples & Mesophilic Bacteria count (cfu/g) & Fungi count $(\mathbf{s f u} / \mathrm{g})$ & E. coli count $(\mathrm{cfu} / \mathrm{g})$ & Coliform count $(\mathrm{cfu} / \mathrm{g})$ \\
\hline $0: 100$ & $4.0 \times 10^{3}$ & $1.5 \times 10^{4}$ & NP & NP \\
\hline $5: 95$ & $2.0 \times 10^{3}$ & $8.0 \times 10^{3}$ & NP & NP \\
\hline $10: 90$ & $1.0 \times 10^{3}$ & $2.1 \times 10^{4}$ & NP & NP \\
\hline $15: 85$ & $1.0 \times 10^{3}$ & $7.0 \times 10^{4}$ & NP & NP \\
\hline $\begin{array}{l}\text { Means fol } \\
\text { Values ar } \\
0: 100=0 \\
5: 95=5 \% \\
10: 90=1( \\
15: 85=1 \\
\text { NP }=\text { Not }\end{array}$ & $\begin{array}{l}\text { ed by the same letter of alphabets in a column } \\
\text { eans of triplicate readings. } \\
\text { F/ 100\% WF, } \\
\text { 9/ } 95 \% \text { WF, } \\
\text { PF/ 90\% WF, } \\
\text { PF/ } 85 \% \mathrm{WF}, \\
\text { sent. }\end{array}$ & significantly different at & & \\
\hline
\end{tabular}

Table 8: Bread quality determination.

\begin{tabular}{llccc}
\hline Samples & Loaf shape & Loaf weight $(\mathbf{g})$ & Loaf volume $\left(\mathbf{c m}^{\mathbf{3}}\right)$ & Specific Loaf Volume $\left(\mathbf{c m}^{\mathbf{3}} / \mathbf{g}\right)$ \\
\hline $0: 100$ & $0.86 \mathrm{a} \pm 0.001$ & $450.00 \mathrm{~b} \pm 2.13$ & $1512.56 \mathrm{a} \pm 0.02$ & $3.36 \mathrm{a} \pm 0.01$ \\
$5: 95$ & $0.67 \mathrm{~b} \pm 0.001$ & $450.00 \mathrm{~b} \pm 2.13$ & $1179.14 \mathrm{c} \pm 0.02$ & $2.62 \mathrm{~b} \pm 0.01$ \\
$10: 90$ & $0.70 \mathrm{~b} \pm 0.001$ & $455.00 \mathrm{~b} \pm 2.13$ & $1171.73 \mathrm{~d} \pm 0.01$ & $2.58 \mathrm{~b} \pm 0.01$ \\
$15: 85$ & $0.69 \mathrm{~b} \pm 0.001$ & $500.00 \mathrm{a} \pm 2.13$ & $1239.84 \mathrm{~b} \pm 0.02$ & $2.48 \mathrm{c} \pm 0.01$ \\
\hline \multicolumn{2}{l}{ Means followed by the same letter of alphabets in a column are not significantly different at $\mathrm{p} \leq 0.05}$. \\
Values are means of triplicate readings. \\
0:100=0\% PF/ 100\% WF, \\
5:95=5\% PF/ 95\% WF, \\
10:90=10\% PF/ 90\% WF, \\
15:85=15\% PF/ 85\% WF.
\end{tabular}


Table 9: Bread quality evaluation.

\begin{tabular}{|c|c|c|c|c|c|c|c|c|c|c|}
\hline Samples & $\begin{array}{l}\text { Crust } \\
\text { colour }\end{array}$ & $\begin{array}{l}\text { Crumb } \\
\text { colour }\end{array}$ & Crumblings & $\begin{array}{l}\text { Crust } \\
\text { holes }\end{array}$ & $\begin{array}{c}\text { Crumb } \\
\text { stability }\end{array}$ & $\begin{array}{c}\text { Reflective surface } \\
\text { in light }\end{array}$ & $\begin{array}{c}\text { Crumb } \\
\text { elasticity }\end{array}$ & Appearance & $\begin{array}{c}\text { Crumb } \\
\text { firmness }\end{array}$ & $\begin{array}{c}\text { Shape } \\
\text { regularity }\end{array}$ \\
\hline $0: 100$ & $1.28 \mathrm{~d}$ & $1.56 \mathrm{~d}$ & $1.96 \mathrm{~d}$ & $2.08 \mathrm{c}$ & $1.92 \mathrm{~b}$ & $1.80 \mathrm{~d}$ & $2.04 \mathrm{c}$ & $1.32 \mathrm{c}$ & $1.96 \mathrm{~d}$ & $1.40 \mathrm{c}$ \\
\hline $5: 95$ & $2.12 \mathrm{c}$ & $2.36 \mathrm{~b}$ & $2.28 b$ & $2.28 \mathrm{~b}$ & $2.16 \mathrm{a}$ & $2.60 \mathrm{~b}$ & $2.56 a$ & $2.44 \mathrm{a}$ & $2.48 \mathrm{a}$ & $2.28 \mathrm{a}$ \\
\hline $10: 90$ & $2.64 \mathrm{a}$ & $2.72 \mathrm{a}$ & $2.56 \mathrm{a}$ & $2.56 \mathrm{a}$ & $2.20 \mathrm{a}$ & $2.84 \mathrm{a}$ & $2.52 \mathrm{a}$ & $2.32 \mathrm{~b}$ & $2.28 \mathrm{~b}$ & $2.08 \mathrm{~b}$ \\
\hline $15: 85$ & $2.28 \mathrm{~b}$ & $2.12 \mathrm{c}$ & $2.04 \mathrm{c}$ & $2.32 \mathrm{~b}$ & $1.96 \mathrm{~b}$ & $2.48 \mathrm{c}$ & $2.36 \mathrm{~b}$ & $2.32 \mathrm{~b}$ & $2.04 \mathrm{c}$ & $2.08 \mathrm{~b}$ \\
\hline
\end{tabular}

Means followed by the same letter of alphabets in a column are not significantly different at $p \leq 0.05$.

Values are means of (number of panelist scores).

$0: 100=0 \% \mathrm{PF} / 100 \% \mathrm{WF}$

$5: 95=5 \% \mathrm{PF} / 95 \% \mathrm{WF}$,

$10: 90=10 \% \mathrm{PF} / 90 \% \mathrm{WF}$

$15: 85=15 \% \mathrm{PF} / 85 \% \mathrm{WF}$. 
Table 10: Sensory evaluation of bread samples.

\begin{tabular}{|c|c|c|c|c|c|}
\hline Samples & Taste & Aroma & Texture & Mouthfeel & Overall acceptability \\
\hline $0: 100$ & $1.68 \mathrm{~d}$ & $2.00 \mathrm{~d}$ & $1.92 \mathrm{c}$ & $1.80 \mathrm{~d}$ & $1.52 \mathrm{~d}$ \\
\hline $5: 95$ & $2.32 b$ & $2.44 \mathrm{~b}$ & $2.04 \mathrm{~b}$ & $2.16 \mathrm{c}$ & $2.28 \mathrm{~b}$ \\
\hline $10: 90$ & $2.52 \mathrm{a}$ & $2.64 \mathrm{a}$ & $2.60 \mathrm{a}$ & $2.60 \mathrm{a}$ & $2.72 \mathrm{a}$ \\
\hline $15: 85$ & $2.00 \mathrm{c}$ & $2.16 \mathrm{c}$ & $2.00 \mathrm{~b}$ & $2.32 b$ & $2.16 \mathrm{c}$ \\
\hline \multicolumn{6}{|c|}{$\begin{array}{l}\text { Means followed by the same letter of alphabets in a column are not significantly different at } \mathrm{p} \leq 0.05 \text {. } \\
\text { Values are means of (number of panelist scores). } \\
0: 100=0 \% \mathrm{PF} / 100 \% \mathrm{WF} \text {, } \\
5: 95=5 \% \mathrm{PF} / 95 \% \mathrm{WF} \\
10: 90=10 \% \mathrm{PF} / 90 \% \mathrm{WF} \\
15: 85=15 \% \mathrm{PF} / 85 \% \mathrm{WF} \text {. }\end{array}$} \\
\hline
\end{tabular}

\section{DISCUSSION}

\section{Chemical composition of flours and bread samples}

\section{Proximate and mineral compositions}

The result shows significant reduction in protein, fat, moisture content and increase in fibre and carbohydrate contents as level of substitution of wheat flour with sweet potato flour increased.

There is significant differences $(\mathrm{p} \leq 0.05)$ in their protein, moisture and carbohydrate content with $100 \%$ / 0\% (PF/WF) having the least value and $0 \% / 100 \%$ (PF/WF) having the highest value of protein, fat and moisture content but in reverse order in their fibre and carbohydrate contents. The proximate composition of the potato substituted bread samples was favourably comparable with the control. This observation is in agreement with the report of Martin (1997), who listed sweet potato as a starchy root crop with high carbohydrate content. It could be inferred from the result that the observation made on the flour samples is in many ways similar to those of the bread produced with the flours. The result shows a considerable significant difference $(p \leq 0.05)$ in all the mineral elements found in the flour samples except for $\mathrm{Cu}, \mathrm{Mg}$ and $\mathrm{Mn}$, where no significant difference was observed. As the level of substitution of wheat flour with sweet potato flour increased, the $\mathrm{Na}$, $\mathrm{K}, \mathrm{P}, \mathrm{Cu}, \mathrm{Mn}$ and $\mathrm{Zn}$ contents also increased but $\mathrm{Ca}, \mathrm{Fe}, \mathrm{Mg}$ and Se contents decreased.
There is significant difference in $\mathrm{Ca}$, $\mathrm{Mg}, \mathrm{P}, \mathrm{K}$ and $\mathrm{Na}$ contents but no significant difference is observed in the remaining mineral elements of the bread samples. It is also inferred that as level of substitution increases, the $\mathrm{Mg}$, and $\mathrm{P}$ contents increases but in reverse order for $\mathrm{Ca}, \mathrm{Fe}$, $\mathrm{cu}$ and $\mathrm{Se}$ contents. However, both macro and micro elements were sufficiently available in the composite bread samples. Besides, the very low content of $\mathrm{Se}$ in both the flours and breads makes the products edible, healthy and ideal for consumption.

\section{Pasting properties of flours}

It is also observed that there is significant difference $(\mathrm{p} \leq 0.05)$ between the substituted flours and all the pasting properties decreased (except for peak time which increased) as the level of substitution increased. This is in accordance with the reports of Pham and Naofumi, (2004) that the final viscosities of the composite flours decreased with the increase in the level of substitution.

\section{Microbiological studies of flour and bread \\ The results obtained here were in accordance with the findings of Onuh and Egwujeh (2005) and Olaoye et al. (2006) who reported that flour and bread with low microbial count would store for a reasonable}


period of time under good storage conditions. No visible growth was recorded for $E$. coli. and Coliform bacteria showing that the water used for all the processes was safe.

\section{Physical properties of bread samples}

The results of determination and evaluation of bread produced from the composite flours are presented in Tables 8 and 9. The higher loaf weights observed in the potato flour substituted samples have positive effect on bread at the retail end. This is in accordance with the findings of Shittu et al. (2007). None of the samples with the control inclusive met the specific volume of $6 \mathrm{~cm}^{3} / \mathrm{g}$ for standard bread. The specific volume of standard bread, according to China Grain Products Research and Development Institute (CGPRDI) should be $6 \mathrm{~cm}^{3} / \mathrm{g}$ and should not be less than $3.5 \mathrm{~cm}^{3} / \mathrm{g}$ (Lin et al., 2009). The lower specific loaf volume of the potatowheat bread samples may be responsible for their higher weights. The Panelists indicated their preference for the potato-wheat bread in terms of the crust colour, crumb holes, stability, elasticity, firmness, shape regularity and appearance as shown in Table 9.

\section{Sensory properties of bread samples}

Table 10 shows the results obtained for the sensory evaluation of the bread samples. When compared with the control sample, no panelist showed a total dislike for the taste of any of the substituted samples, implying that the taste was not affected by the level of substitution. In fact the sweet potato flour improved the sensory properties of the bread produced judging by the result of the sensory evaluation by the panelists. The $10 \%$ sweet potato inclusion was rated best.

\section{Conclusion}

The results obtained from this study have demonstrated the potential for the production of bread from sweet potato and wheat composite flour. The wheat flour could be substituted with sweet potato flour up to $15 \%$ level of substitution without adverse effect on the taste, appearance, loaf weight and volume, crumb colour, firmness and shape regularity of the bread produced. This therefore implies that substituting wheat flour with potato flour could be beneficial to man through improvement of the physicochemical and sensory qualities of such products.

\section{REFERENCES}

Ade-Omowaye BIO, Bolarinwa BA, Adebiyi AO. 2008. Evaluation of Tigernut (Cyperus esculentus)- wheat composite flour and bread. African Journal of Food Science, 2: 087-089.

Akobundu ENT. 2006. Breadmaking technology and Ingredients for bread making. A paper presented at a training workshop on the use of cassava/wheat composite flour and non bromate additives for making bread and other confectioneries. Held at Michael Okpara University of Agriculture, Umudike, 10 12 October, 2006.

American Association of Cereal Chemists. 2000. Approved Methods of the AACC ( $10^{\text {th }}$ edition), St Paul MN. AACC.

Association of Official Analytical Chemists. 1990. Official Methods of Analysis (15 ${ }^{\text {th }}$ Edition). AOAC: Arlington, Virginia, USA. 2; 142- 202.

Edema MO, Sanni LO, Sanni AI. 2005. Evaluation of maize-soybean flour blends for sour maize bread production in Nigeria. Afric. J. Biotechnol., 4(9): 911 918.

Eddy NO, Udofia PG, Eyo D. 2007. Sensory Evaluation of Wheat/Cassava composite bread and effect of label information on acceptance and preference. African Journal of Biotechnology, 6(20): 2415 2418.

Giami GY, Amasisi T, Ekiyor G. 2004. Comparison of bread making properties 
of composite flour from kernels or roasted and boiled African bread fruit (Treculia Africana) seed. J. Mat. Res., 1 (1): 16-25.

Iheagwara MC, Iwuoha CI. 2005. Evaluation of Physiochemical Properties of Sweet Potato (Ipomea batatas L.) Flour as affected by Tuber Section and Heat Treatment. NIFST AGM, 11- 13 October, 2005: 264- 265.

Lin L, Liu H, Yu Y, Lin SD, Mau J. 2009. Quality and antioxidant property of buckwheat enhanced wheat bread. Food Chemistry. 37: 461- 467.

Malomo SA. 2010. Effect of partial substitution of wheat flour with breadfruit and Breadnut flour on bread quality and Consumer Perception. M. Tech. Thesis. Food Science and Technology, Department, Federal University of Technology, Akure, Nigeria.

Malomo SA, Eleyinmi AF, Fashakin JB. 2011. Chemical Composition, Rheological Properties and Breadmaking Potentials of Composite Flours from Breadfruit, Breadnut and Wheat. African Journal of Food Science (In press).

Martin FW. 1997. Techniques and Problems in Small- scale Production of Flour from Sweet Potato. J. Agric. Univ. Puer to Rico, 3: 423- 432.

Olaoye OA, Onilude AA, Idowu OA. 2006. Quality characteristic of bread producedMartin FW. 1997. Techniques and Problems in Small- scale Production of Flour from Sweet Potato. J. Agric. Univ. Puer to Rico, 3: 423- 432.
Olaoye OA, Onilude AA, Idowu OA. 2006. Quality characteristic of bread produced from wheat, plantain and soybeans flour. Afri. J. Food Biotechnol., 5(11): 1102 - 1106.

Oluwole OB, Oke OU, Oyegbami FO, Adeyoju AO, Adetuyi DO, Olapade AA, Derenle A, Ozumba AU. 2005. Swelling Profile of Processed Sweet Poatato Flour. NIFST AGM, 11-13 October, 2005: 266- 267.

Onuh JO, Egwujeh SID. 2005. Sensory and Microbial Quality of Stored Sweet Potato (Ipomea batatas L.) Flour. NIFST Proceedings of the $29^{\text {th }}$ Annual Conference, 11- 13 October, 2005: 284285.

Pham VH, Naofumi MO. 2004. Dough properties and bread quality of flour supplemented with cross linked corn starches. Food Research International, 37, 461-467.

Pomeranz Y, Clifton EM. 1996. Food Analysis: Theory and Practice $\left(3^{\text {rd }}\right.$ Edition). CBS Publishers and Distributors: India; 45- 56.

Shittu TA, Raji AO, Sanni LO. 2007. Bread from composite cassava-wheat flour: effect of baking time and temperatures on some physical properties of bread loaf. Food Research International, 40: 280- 290. 